\title{
Fever, headache, fatigue and chancre in a traveller returning from Tanzania
}

\author{
JP Davis MD PhD ${ }^{1 *}$, VP Chaubey MDCM ${ }^{1,2 *}$, R Warren BA LLB MD ${ }^{3}, M$ Parkins MD MSc ${ }^{1,2}, M$ Louie $\mathrm{MD}^{1,2,4}$, \\ D Gregson $\mathrm{MD}^{1,2,5}$, D Sabuda BSc BSP6, S Kuhn MD MSc ${ }^{1,7}$
}

\section{CASE PRESENTATION}

A previously healthy 52-year-old man presented in January 2009 with $48 \mathrm{~h}$ history of fever, headache, nonpurulent conjunctivitis, fatigue and a left subclavicular chancre. His symptoms began as he was returning from a three-week trip to East Africa during which he ascended Mount Kilimanjaro, visited the Ngorongoro crater, the Serengeti and a resort in Zanzibar. He denied any insect bites or exposure to fresh water. He had suffered a sunburn in Zanzibar and, over four days, noticed the evolution of a left subclavicular chancre. He had received pretravel counselling and had accepted all relevant vaccinations and was compliant with his malaria prophylaxis. He denied any HIV risk factors.

On presentation, the patient was febrile $\left(40.6^{\circ} \mathrm{C}\right)$, but his other vital signs were within normal limits. A sunburn was noted on his chest, as well as a $4 \mathrm{~cm} \times 3 \mathrm{~cm}$ chancre below his left clavicle (Figure 1). This lesion was indurated, nontender and nonfluctuant. A physical examination and laboratory investigations were otherwise unremarkable. A diagnostic test was performed.

\section{Diagnosis}

Thin blood smears performed to rule out malaria revealed the presence of a single trypanosome (Figure 2), which established a diagnosis of East African trypanosomiasis.

\section{DISCUSSION}

The differential diagnosis of fever and chancre/eschar in a returning traveller includes, in addition to human African trypanosomiasis (HAT): tularemia (Francisella tularensis), African tick bite fever (Rickettsia africanae), scrub typhus (Orientia tsutsugamushi), plague (Yersinia pestis) and cutaneous anthrax (Bacillus anthracis). However, trypanosomiasis produces a true chancre whereas several of the other diseases produce eschars, but these are often difficult to distinguish. The chancre represents primary infection at the point of inoculation by the vector. Scrub typhus can be ruled out because this disease is restricted to Southeast Asia and South Asia. The buboes of plague can rupture and evolve to resemble a chancre/eschar, but are generally found in regions where lymph nodes are distributed. The necrotic lesion associated with anthrax can be indistinguishable from a chancre/eschar (1). Other typical, febrile, travel-related infections must also be considered. HAT is caused by the flagellated protozoan parasite Trypanosoma brucei and is transmitted by the tsetse fly (Glossina species).

There are two forms of the disease that are caused by different subspecies of $T$ brucei $(T b)$ : West African (Tb gambiense) and East African (Tb rhodesiense). Although the names reflect their geographical distribution, there are regions of overlap. Both forms are fatal without treatment (2). HAT is a very rare diagnosis in returning travellers (3), although occasional outbreaks have been reported (4). Although West African trypanosomiasis has been reported (5), to our knowledge, this is the first case of East African trypanosomiasis imported to Canada.

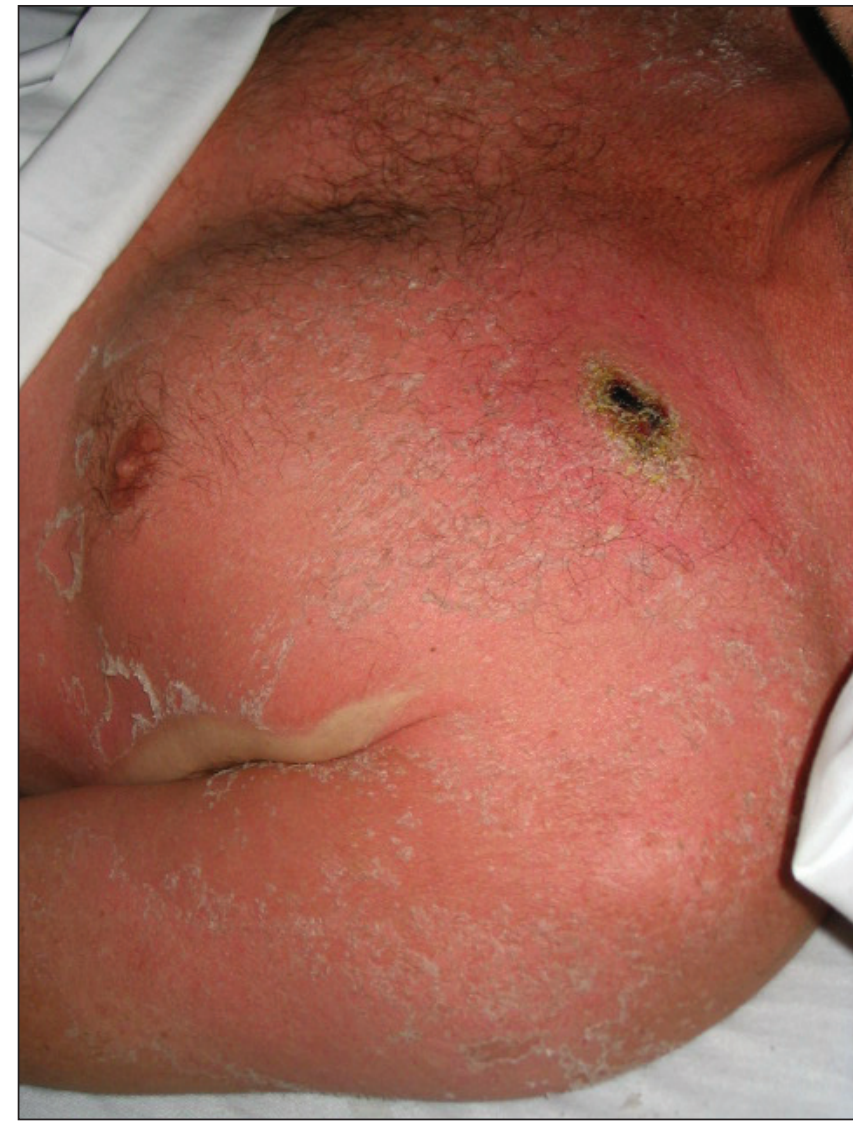

Figure 1) Chancre associated with East African trypanosomiasis

The natural course of HAT has two stages: the early stage in which the protozoa proliferate and spread through the blood and lymphatic system; and the late stage, in which the parasites cross the blood brain barrier and cause a progressive, diffuse meningoencephalitis. The two forms differ in the rate of progression of the disease. Tb gambiense is a more indolent infection that may be asymptomatic for months to years, while $\mathrm{Tb}$ rhodesiense progresses over the course of weeks. Symptoms of HAT during the early stage include fever, headache, malaise, rash, swelling and generalized body pain. The second stage of the disease can produce a myriad of neurological symptoms, including the classic day-night reversal, hence the common name 'sleeping sickness'. Signs of trypanosomiasis on physical examination may include lymphadenopathy and organomegaly (more common with Tb gambiense) and chancre (more common with Tb rhodesiense). Leukocytosis,

*These authors contributed equally to the manuscript

${ }^{1}$ Department of Medicine; ${ }^{2}$ Department of Microbiology and Infectious Diseases, University of Calgary, Calgary; ${ }^{3}$ Rural Alberta North Residency

Training Program, University of Alberta, Edmonton; ${ }^{4}$ Provincial Laboratory for Public Health, Alberta Health Services; ${ }^{5}$ Calgary Laboratory

Services, Alberta Health Services; ${ }^{6}$ Department of Pharmacy Services, Alberta Health Services, Calgary, Alberta; ${ }^{7}$ Department of Pediatrics,

University of Calgary

Correspondence: Dr Vikas Pankaj Chaubey, University of Calgary, Room 303, 3rd Floor North Tower, 1403-29th Street Northwest, Calgary,

Alberta T2N 2T9. Telephone 403-944-2325, e-mail vikas.chaubey@albertahealthservices.ca 
leukopenia, anemia, thrombocytopenia, elevated hepatic transaminases, coagulation abnormalities and azotemia can also be observed.

The diagnosis of HAT is difficult because of nonspecific symptoms and the lack of sensitivity of the diagnostic tests. Patients with East African trypanosomiasis generally present with high-grade parasitemia. The West African form is often associated with low-grade parasitemia; therefore, serological or polymerase chain reaction testing must be undertaken to confirm the diagnosis (1). A definitive diagnosis of trypanosomiasis requires demonstration of the parasite in blood, tissue (lymph node or chancre) or cerebrospinal fluid (CSF). Lumbar puncture is indicated even in the absence of neurological symptoms because the drug choice for treatment is dependent on the stage of the disease. The lumbar puncture is typically delayed until at least one dose of therapy has been administered to avoid the theoretical risk of carrying the parasite through the blood brain barrier. A positive CSF sample is defined as having a white blood cell count of $70.5 \times 10^{9} / \mathrm{L}$, or increased protein, increased immunoglobulin $\mathrm{M}$ level or the presence of protozoa (6).

Treatment options for $T b$ rhodesiense infection in the early and late stages are suramin and melarsoprol, respectively $(6,7)$. Suramin (available through Health Canada's Special Access Program <www. hc-sc.gc.ca/dhp-mps/acces/drugs-drogues/index-eng.php $>$ ) has a long list of side effects, notably, anaphylaxis, renal dysfunction, neurological toxicity and rash. The side effects of melarsoprol (provided by the WHO via Health Canada's Special Access Program) are also dramatic and occur in quite high frequencies, including death $(5 \%)$, reactive encephalopathy (10\%), and hepatic and renal toxicity, as well as peripheral neuropathy. For Tb gambiense infection, treatment consists of pentamidine for the early stage and eflornithine or melarsoprol for the late stage (7). Treatment protocols are available from the Centers for Disease Control (Atlanta, Georgia, USA), <www. dpd.cdc.gov/dpdx/html/trypanosomiasisafrican.htm>. It is recommended that blood and CSF analysis be performed at three, six, 12, 18 and 24 months post-treatment (8). This intense and invasive follow-up is more important in cases of Tb gambiense to ensure eradication of the parasite.

\section{Case follow-up}

The patient was admitted to hospital where he developed thrombocytopenia requiring platelet transfusion. Treatment with suramin was initiated and, within $48 \mathrm{~h}$, the patient's condition had improved markedly. Lumbar puncture was subsequently performed and was unremarkable. The patient received five doses of suramin, a total of $1.5 \mathrm{~g}(16 \mathrm{mg} / \mathrm{kg})$ on days 1, 3, 7, 14 and 21. Adverse effects included nonspecific rash, elevated liver enzyme levels and nephrotic range proteinuria. These side effects were transient, but 12 months later, the patient was still affected by modest chronic renal dysfunction (creatinine level $135 \mu \mathrm{mol} / \mathrm{L}$ ). CSF analysis performed three months post-treatment was unremarkable, and the patient refused to undergo additional lumbar punctures.

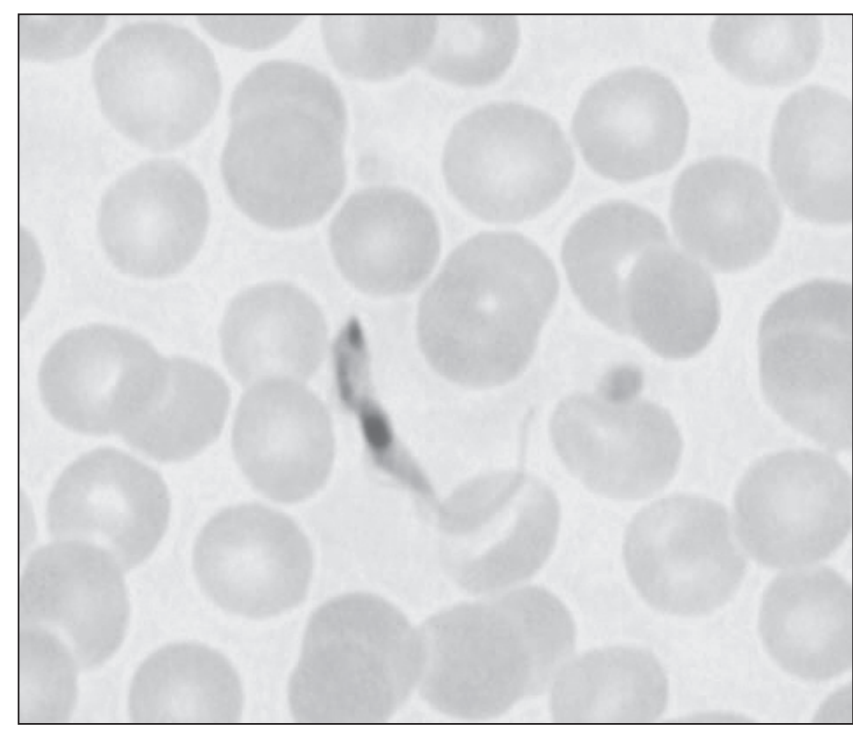

Figure 2) A single trypanosome identified by microscopy

DISCLOSURES: The authors declare no competing interests relevant to this article.

\section{REFERENCES}

1. Mawhorter SD, Longworth DL. Cutaneous Lesions. In: Guerrant RL, Walker DH, Weller PF, eds. Tropical Infectious Diseases: Principles, Pathogens, and Practice, 3rd edn. Philadelphia: Saunders Elsevier, 2011:950-974.

2. Kirchhoff, LV (2009). Agents of African Trypanosomiasis (sleeping sickness). In: Mandell GL, Bennett JE, Dolin R, eds. Mandell, Douglas and Bennett's Principles and Practices of Infectious Diseases, 7 th edn. Philadelphia: Churchill Livingstone Elsevier, 2009:3489-94.

3. Sinha A, Grace C, Alston WK, et al. Trypanosomiasis in two travelers from the United States. Clin Inf Dis 1999;29:840-4.

4. Jelinek T, Bisoffi Z, Bonazzi L, et al. Cluster of African trypanosomiasis in travelers to Tanzanian national parks. Emerg Inf Dis 2002;8;634-5.

5. Seah SKK, Flegel KM. African trypanosomiasis in Canada. CMAJ 1972;106:902-3.

6. Lejon V, Bushcer P. Cerebrospinal fluid in human African trypanosomiasis: A key to diagnosis, therapeutic decision and posttreatment follow-up. Trop Med Int Health 2005;10:395-403.

7. Legros D, Ollivier G, Gastellu-Etchegorry, et al. Treatment of human African trypanosomiasis - present situation and needs for research and development. Lancet Inf Dis 2002;2:437-40.

8. World Health Organization: Control and Surveillance of African Trypanosomiasis. WHO Technical Report Series 1998; \#881. 


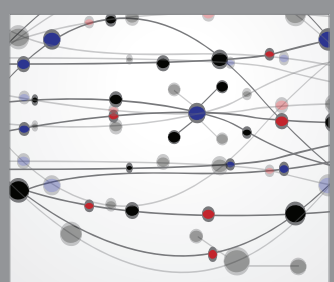

The Scientific World Journal
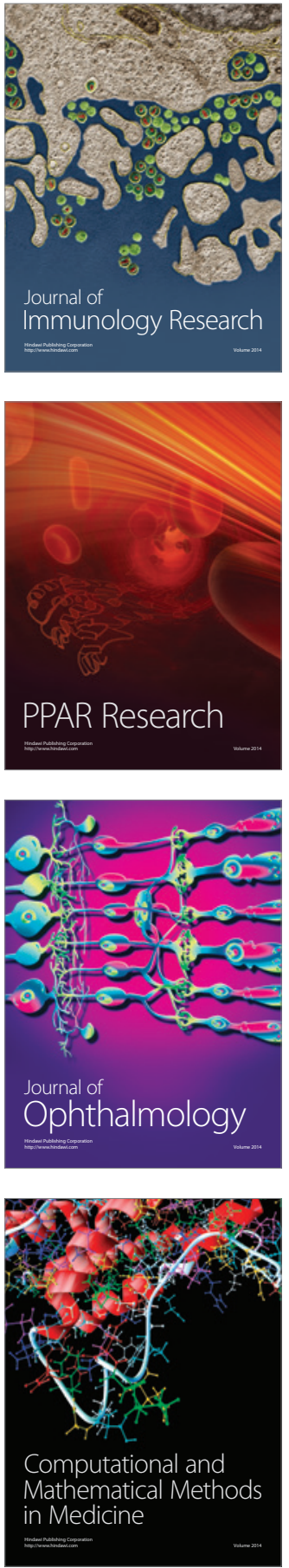

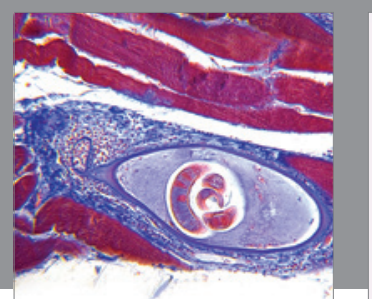

Gastroenterology Research and Practice

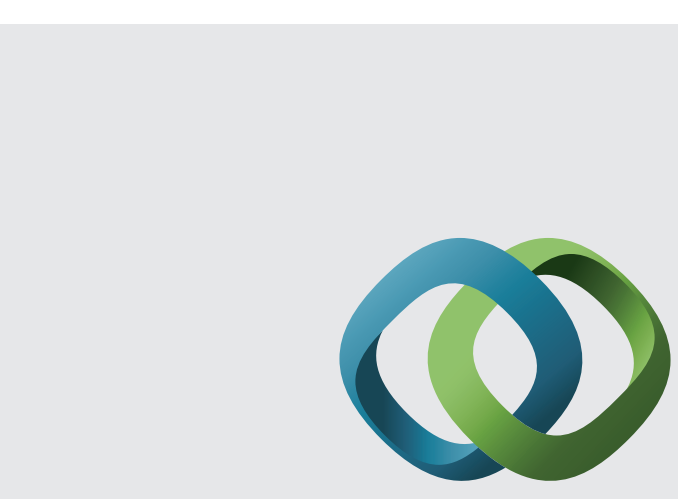

\section{Hindawi}

Submit your manuscripts at

http://www.hindawi.com
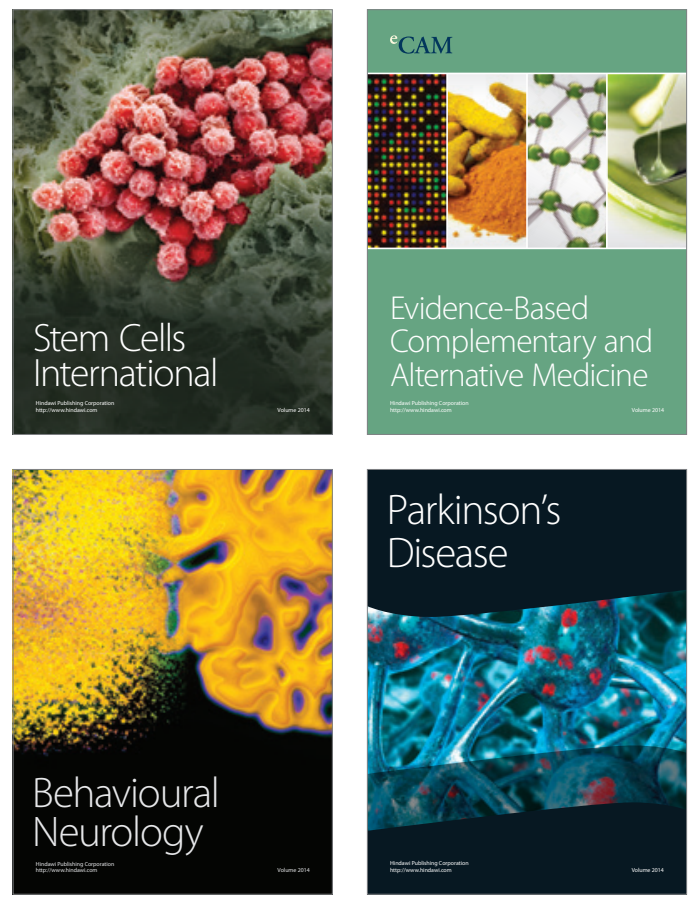
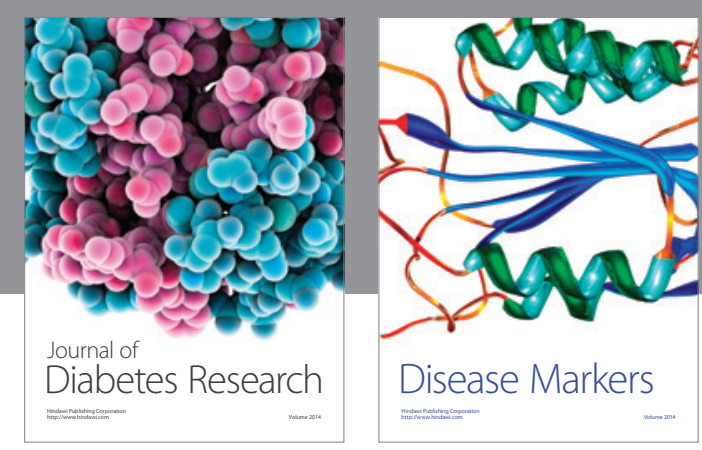

Disease Markers
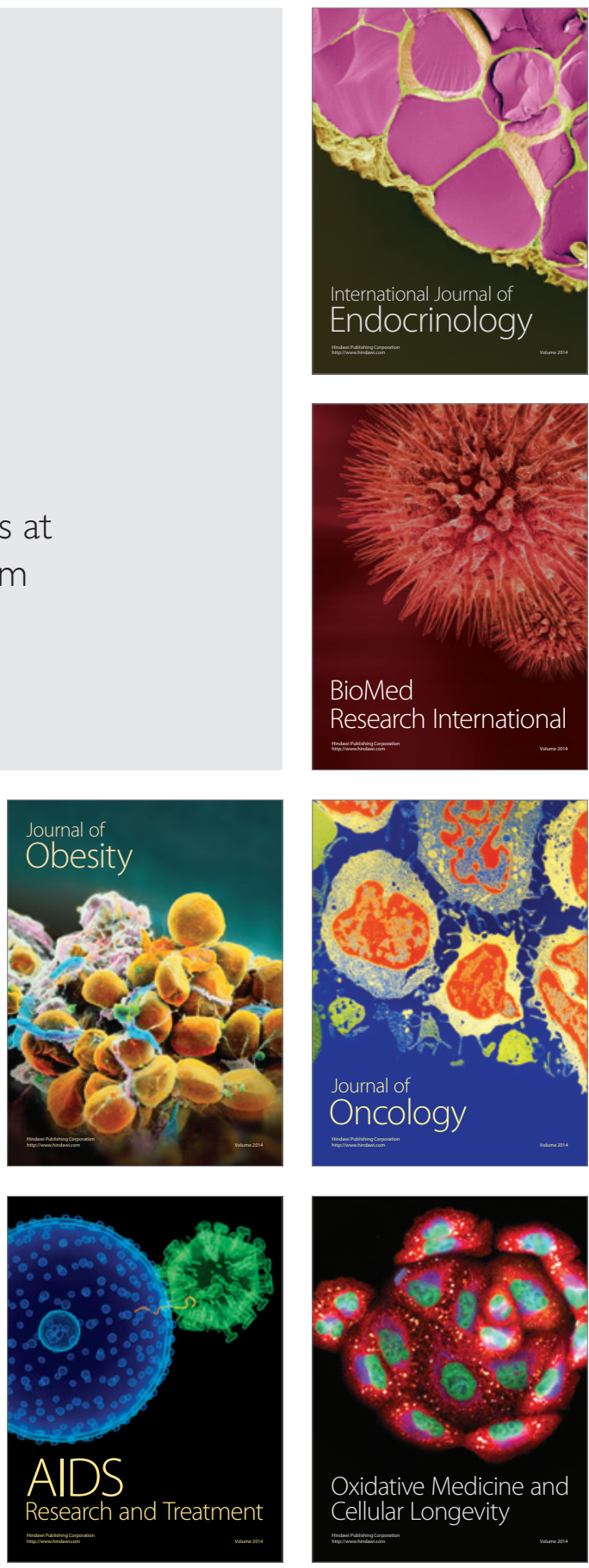